\title{
BMJ Open Association between gaps in antihypertensive medication adherence and injurious falls in older community- dwelling adults: a prospective cohort study
}

\author{
Paul Dillon, ${ }^{1}$ Susan M Smith, ${ }^{2}$ Paul John Gallagher, ${ }^{1}$ Gráinne Cousins ${ }^{1}$
}

To cite: Dillon P, Smith SM, Gallagher PJ, et al. Association between gaps in antihypertensive medication adherence and injurious falls in older communitydwelling adults: a prospective cohort study. BMJ Open 2019;9:e022927. doi:10.1136/ bmjopen-2018-022927

- Prepublication history for this paper is available online. To view these files please visit the journal online (http://dx.doi org/10.1136/bmjopen-2018022927).

Received 14 March 2018 Revised 25 0ctober 2018 Accepted 5 November 2018

Check for updates

(C) Author(s) (or their employer(s)) 2019. Re-use permitted under CC BY-NC. No commercial re-use. See rights and permissions. Published by BMJ.

${ }^{1}$ School of Pharmacy, Royal College of Surgeons in Ireland, Dublin, Ireland

${ }^{2}$ Department of General Practice, HRB Centre for Primary Care Research, Royal College of Surgeons in Ireland, Dublin, Ireland

Correspondence to

Paul Dillon; pauldillon@rcsi.ie

\section{ABSTRACT}

Objective Growing evidence suggests that older adults are at an increased risk of injurious falls when initiating antihypertensive medication, while the evidence regarding long-term use of antihypertensive medication and the risk of falling is mixed. However, long-term users who stop and start these medications may have a similar risk of falling to initial users of antihypertensive medication. Our aim was to evaluate the association between gaps in antihypertensive medication adherence and injurious falls in older ( $\geq 65$ years) community-dwelling, long-term ( $\geq \geq 1$ year) antihypertensive users.

Design Prospective cohort study.

Setting Irish Community Pharmacy.

Participants Consecutive participants presenting a prescription for antihypertensive medication to 106 community pharmacies nationwide, community-dwelling, $\geq 65$ years, with no evidence of cognitive impairment, taking antihypertensive medication for $\geq 1$ year $(n=938)$. Measures Gaps in antihypertensive medication adherence were evaluated from linked dispensing records as the number of 5-day gaps between sequential supplies over the 12-month period prior to baseline. Injurious falls during follow-up were recorded via questionnaire during structured telephone interviews at 12 months.

Results At 12 months, $8.1 \%(n=76)$ of participants reported an injurious fall requiring medical attention. The mean number of 5 -day gaps in medication refill behaviour was 1.47 (SD 1.58). In adjusted, modified Poisson models, 5-day medication refill gaps at baseline were associated with a higher risk of an injurious fall during follow-up (aRR $1.18,95 \% \mathrm{Cl} 1.02$ to $1.37, p=0.024)$.

Conclusion Each 5-day gap in antihypertensive refill adherence increased the risk of self-reported injurious falls by $18 \%$. Gaps in antihypertensive adherence may be a marker for increased risk of injurious falls. It is unknown whether adherence-interventions will reduce subsequent risk. This finding is hypothesis generating and should be replicated in similar populations.

\section{INTRODUCTION}

While many studies have demonstrated the benefits of antihypertensive agents on
Strengths and limitations of this study

- Prospective cohort study of a community-dwelling older adults recruited from community pharmacies across Ireland.

- Objective evaluation of medication exposures and medication adherence although injurious falls were self-reported which may lead to misclassification.

- Negative control exposure analyses to account for a potential healthy adherer effect were conducted.

- Data on some important confounders, such as history of previous falls, frailty and disability, and the date of falls were not available to us.

myocardial infarction and stroke risk reduction, ${ }^{1-3}$ concerns have been raised regarding the risk of falls associated with antihypertensive medications among older adults. ${ }^{4}$ Growing evidence suggests that the associated risk of injurious falls varies according to the duration of treatment. ${ }^{5}{ }^{6}$ Studies have consistently shown that older adults are at a greater risk for injurious falls or hip fractures shortly after initiating antihypertensive medications. ${ }^{7-12}$ Although the underlying mechanism is unknown, it is thought that antihypertensive medication cause or exacerbate orthostatic hypotension in the elderly resulting in poor balance, weakness, dizziness and falls. ${ }^{5}$ The prevalence of orthostatic hypotension increases with age and uncontrolled hypertension, ${ }^{13}$ and orthostatic hypotension itself is associated with an increased risk of injurious falls. ${ }^{1415}$

Observational studies investigating the risk of injurious falls among long-term users of antihypertensive agents have yielded mixed results. ${ }^{16-23}$ Despite the known risks associated with treatment initiation, studies of longterm users have not considered the potential effect of medication adherence, specifically 
the quality of daily dose-taking behaviour of patients, known as dose implementation, ${ }^{24}$ on the risk of injurious falls. It is plausible that failure to consistently take antihypertensive medications may result in fluctuations in blood pressure, which could lead to an increased risk of falls. ${ }^{25}$ A study of electronically compiled dosing histories revealed intervals between antihypertensive doses to be prevalent. On any given day, $10 \%$ of doses were omitted, with $43 \%$ of missed doses occurring during a sequence of $\geq 3$ days of missed doses. Overall, approximately half of patients omit doses for $\geq 3$ days at least once a year. ${ }^{26}$ The quality of implementation of a medication regimen should be interpreted according to the pharmacology of the drug. During gaps in antihypertensive medication use, the pharmacological effects on blood pressure gradually diminish, ${ }^{27-29}$ and on resumption of therapy, patients may experience acute changes to blood pressure similar to initial use.

Our aim was to evaluate medication adherence, specifically as the quality of dose implementation with long-term ( $\geq 1$ year) antihypertensive medication use, and test its association with injurious falls in a cohort of community-dwelling older adults ( $\geq 65$ years).

\section{METHODS}

\section{Study setting and design}

We conducted a prospective cohort study, recruiting participants from 106 community pharmacies across the Republic of Ireland between March and May 2014. Pharmacies were selected on the basis of participating in the National Pharmacy Internship Programme (NPIP). Each pharmacy recruited 15 consecutive participants presenting with a prescription for at least one medication for hypertension, aged $\geq 65$ years, community-dwelling, able to speak and understand English with no evidence of cognitive impairment as judged by the pharmacist. In total, 2231 consecutive patients were invited to participate at baseline, $1592(71.4 \%)$ consented to complete the baseline telephone interview and to link their pharmacy dispensing records. Participants completed a structured telephone interview with trained pharmacy interns at baseline and 12 months. This consisted of structured questionnaires at baseline and 12 months measuring participant sociodemographics, medical history and falls, based on similar questionnaires included in longitudinal studies such as the Irish Longitudinal Study of Ageing (TILDA) ${ }^{30}$ Pharmacy interns were trained to undertake the structured telephone interviews and completed the interviews using standardised data collection forms. The data collection forms included templates for recording the date and time of telephone calls made to participants and the structured interview. Interviews were subsequently linked to each patients' pharmacy records.

We evaluated medication adherence as the quality of dose implementation using pharmacy records for the 12 months prior to baseline, with 5-day gaps in prescription-refill flagged as poor dose implementation. Injurious

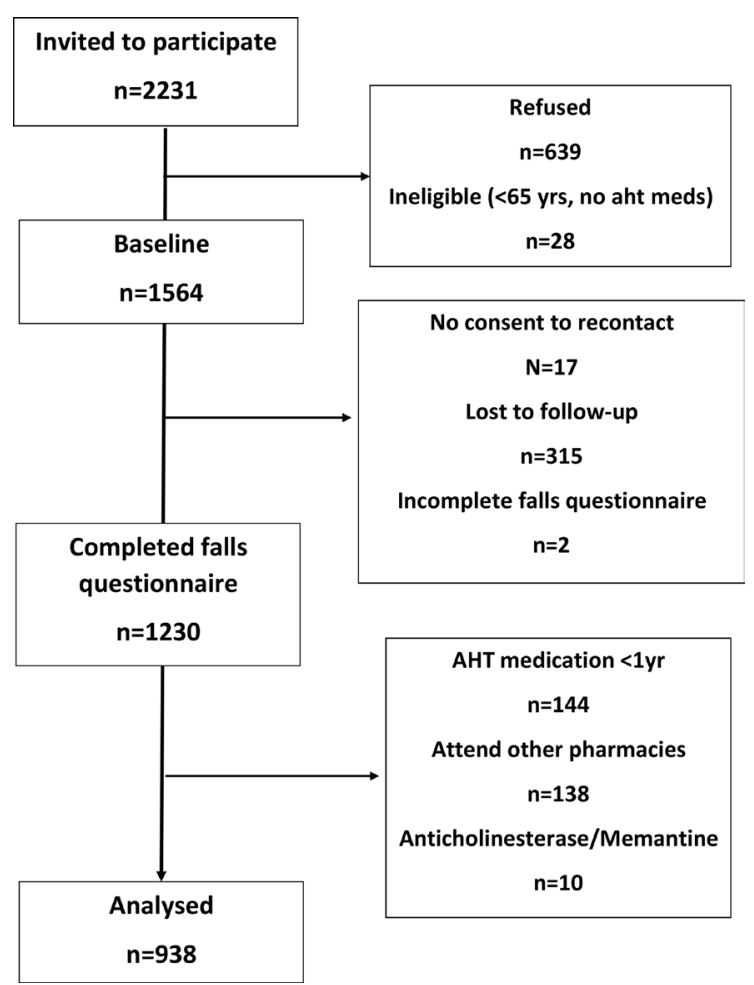

Figure 1 Flow of participants through the study. $\mathrm{AHT}$, antihypertensive.

falls were assessed via self-report at 12 month interview. Participants provided written informed consent. Ethical approval for this study was granted by the Research Ethics Committee of the Royal College of Surgeons in Ireland.

\section{Patient involvement}

No patients were involved in this study.

\section{Inclusion/exclusion criteria}

Participants completing the falls questionnaire at the follow-up interview $(n=1230)$ were considered for inclusion. Subsequently, we excluded participants reporting the use of antihypertensive medication for $<12$ months and participants attending other pharmacies as their pharmacy records were not complete. Participants who were dispensed anticholinesterase medication or memantine during the study period, a proxy indicator of cognitive impairment, were also excluded due to poor recall of falls and associations with lower medication adherence, ${ }^{31-33}$ leaving a final sample of 938 participants. Figure 1 outlines the flow of participants through the study.

\section{Outcome}

Injurious falls were assessed during the 12-month follow-up period (April 2014-May 2015). At 12-month follow-up interview, we asked participants, 'Have you fallen in the last year?' and 'Did you injure yourself seriously enough to need medical treatment'. A standard definition of a fall as 'an unexpected event in which participants come to rest on the ground, floor or lower level' was used. ${ }^{34}$ 
Injurious falls are likely to be recalled more accurately due to the subsequent medical treatment. ${ }^{31}$

\section{Exposure}

We evaluated medication adherence, specifically the quality of dose implementation for the 12-month period prior to baseline (March 2013-April 2014), by measuring the number of 5-day gaps in prescription refill from linked patient pharmacy records. Pharmacy dispensing records are an objective and indirect measure of medication adherence. ${ }^{35}{ }^{36}$ We chose a 5-day gap based on the literature describing the gradual decline of the antihypertensive medication effect on blood pressure over 3 days, ${ }^{27-29}$ extending this period to 5 days due to the indirect method of adherence measurement, and to allow for potential variation in antihypertensive medication pharmacology. ${ }^{37}$ The number of occasions when gaps of 5 days occurred between sequential supplies during the previous 12 months was evaluated at baseline. Oversupplies were credited to a maximum excess of 180 days of medication.

\section{Covariates}

Confounding factors were chosen based on known associations with falls and medication adherence behaviour. Age, gender, marital status, education level and comorbidities including depression, diabetes mellitus, rheumatoid arthritis and stroke were recorded at the baseline interview. ${ }^{33}$ Parkinson's disease and urinary incontinence were identified by proxy from medication (ATC N04; ATC G04BD) ${ }^{33} \mathrm{~A}$ meta-analysis by Woolcott $e t$ al was used to classify medication as falls risk increasing drugs (antipsychotics, antidepressants, benzodiazepines, non-steroidal anti-inflammatories, opiates and sedatives) from linked dispensing records. ${ }^{38}$ The number of regular medicines dispensed may also be associated with an increased falls risk. ${ }^{22}$ Class of antihypertensive used may affect falls risk, for example, ACE inhibitors and angiotensin II receptor blockers have been observed to lower the risk of falls. ${ }^{16}{ }^{19}$ Moderate ${ }^{17}$ and high ${ }^{20}$ doses have also been linked to an increased falls risk. Standardised doses of antihypertensive medication were determined using the WHO's daily defined dose (WHO-DDD). Addition and titration of antihypertensive medication may precipitate a fall, ${ }^{11}$ and a binary variable was created to account for this during follow-up.

\section{Statistical analysis}

Descriptive statistics are presented for participant characteristics at both baseline and follow-up. Means and SD are presented for continuous variables, whereas counts and proportions for categorical variables. The association between 5-day gaps in medication refill and injurious falls during follow-up was estimated using modified Poisson regression to obtain relative risks rather than ORs, which is considered more suitable when outcomes are not rare. ${ }^{39}$ Standard errors were adjusted in regression models using the Sandwich estimator, due to the potential for the dependency of observations at the pharmacy level. Rather than selecting confounding factors for inclusion in the final model based on univariate associations, the final multivariable model was adjusted for all measured confounders.

\section{Sensitivity analysis}

Due to concerns of multivariate regression models with many covariates and a low number of outcome events, we also undertook a sensitivity analysis using a propensity score covariate adjustment model. To reduce the number of confounders, we estimated a Poisson model with 5-day gaps in antihypertensive prescription refills as the outcome and all other covariates as predictors. The predicted value from the resultant regression equation for each observation was then used to adjust for covariates in the final modified Poisson regression model with injurious falls as the outcome and number of 5-day gaps in antihypertensive prescription refill as the predictor variable. ${ }^{40}$

\section{Negative control analysis}

Finally, a negative control exposure model was also estimated. Negative controls are a tool for detecting confounding bias in observational studies to help identify potential non-causal associations. ${ }^{41}$ In negative control tests, conditions are reproduced that cannot involve the hypothesised causal mechanism, but likely involve the same sources of bias, such as the healthy adherer bias in adherence research. ${ }^{41} 42$ Patients with poorer medication adherence tend to have worse outcomes, leading to spurious associations in adherence research known as the healthy adherer bias. ${ }^{42}$ Negative control exposure models, in particular, are useful to detect confounding resulting from the healthy adherer bias, due to the ability to change the conditions by choosing an alternative medication to evaluate adherence that removes the hypothesised causal mechanism, but maintaining the potential for the healthy adherer bias. In the current study, the association between 5-day gaps in medication-taking behaviour to antithrombotic medication and injurious falls was also estimated. Antithrombotics (ATC Code B01AC, B01AE, B01AF, eg, aspirin, dabigatran and rivaroxaban) were chosen due to the high prevalence of use in this sample and the lack of a theoretical association with falls. An association between gaps in antithrombotic medication adherence and injurious falls would indicate the presence of confounding associated with the exposure variable. ${ }^{43}$ The characteristics of the subsample may differ statistically from the entire sample $(n=938)$ and introduce bias into the estimates of the negative control analysis. Differences in participant characteristics between those using antithrombotic and those not using antithrombotic medication were thus also evaluated using Pearsons' $\chi^{2}$ and $t$-tests. Based on these differences, an additional weighted negative control regression analysis using inverse probability weights was undertaken. Weights were estimated as the ratio of the predicted probabilities using two probit regressions, one with and one without statistically different participant characteristic variables.

Statistical modelling was performed using Stata V.14. 


\section{RESULTS}

The mean age of this sample was 76.1 years and $47.9 \%$ were men $(n=938)$. The mean duration of antihypertensive therapy was 11.7 years with participants taking a mean of 2.1 different classes of antihypertensive medication. Participants reported a mean of 2.4 additional comorbid conditions and were taking on average 6.2 regular medication. Table 1 provides a summary of participant characteristics at the baseline interview and 12-month follow-up.

During the 12-month follow-up, 8.1\% ( $\mathrm{n}=76)$ of participants reported an injurious fall requiring medical attention or treatment. At baseline, the mean number of 5-day gaps in medication refill behaviour was 1.47 (SD 1.58).

\section{Primary analysis}

Table 2 details the results of the modified Poisson regression model. In the adjusted analysis, 5-day medication refill gaps at baseline were prospectively associated with a higher risk of an injurious fall during follow-up (aRR 1.18, 95\% CI 1.02 to $1.37, p=0.024$ ). As demonstrated in figure 2 , each 5-day gap in antihypertensive medication refill adherence was associated with an $18 \%$ increased risk of a self-reported injurious fall during follow-up. For participants with one 5-day gap in medication refill compared with participants with none, the respective absolute adjusted risk of an injurious fall is $6.9 \%$ vs 5.8\%, equating to an additional 11 injurious falls per 1000 patients with one 5-day gap in medication refill adherence.

\section{Sensitivity analyses}

The propensity score adjustment model analysis $(\mathrm{n}=724)$ used a propensity score covariate adjustment method to control for covariates listed in table 1 . The propensity score covariate adjustment model produced similar estimates (aRR $1.17,95 \%$ CI 1.03 to $1.35, p=0.020$ ) to our primary analysis.

\section{Negative control analysis}

For the negative control exposure analysis, $60 \% \quad(n=566)$ of participants were regularly using antithrombotic medication. The mean number of 5-day gaps in antithrombotic prescription refill was 1.28 ( $S D$ 1.40). Table 3 presents the results of the negative control analysis. In multivariate modified Poisson regression analysis, adjusted for covariates, 5-day gaps in antithrombotic medication refill behaviour were not associated with injurious falls (aRR $1.04,95 \%$ CI 0.84 to $1.28, p=0.728)$. In comparison with the antihypertensive sample $(\mathrm{n}=938)$, the antithrombotic sample consisted of a statistically significant higher proportion of men, a higher rate of comorbidities and higher rate of regular medication use. To adjust for potential bias, this subsample of 566 participants was re-weighted using inverse probability weights, estimated as the ratio of the predicted probabilities using two probit regressions, one with and one without statistically significant variables (gender, comoribidites and regular medication use). In weighted multivariate modified Poisson regression analysis, similar estimates were observed. Adjusting for covariates, 5-day gaps in antithrombotic
Table 1 A summary of participant characteristics as evaluated at the baseline interview and follow-up via questionnaire and via linked dispensing records $(n=938)$

\begin{tabular}{|c|c|c|}
\hline & Baseline & Follow-up \\
\hline \multicolumn{3}{|l|}{ Demographics } \\
\hline Age, mean (SD) & $76.1(6.1)$ & $77.1(6.1)$ \\
\hline Male, \% (n) & $47.9(449)$ & $47.9(449)$ \\
\hline Year on AHT meds, mean (SD) & $11.7(8.9)$ & $12.7(8.9)$ \\
\hline \multicolumn{3}{|l|}{ Education } \\
\hline Primary, \% (n) & $26.7(250)$ & $26.7(250)$ \\
\hline Secondary, \% (n) & $42.0(394)$ & $42.0(394)$ \\
\hline Third level, \% (n) & $26.4(248)$ & $26.4(248)$ \\
\hline \multicolumn{3}{|l|}{ Marital status } \\
\hline Married/partner, \% (n) & $59.3(556)$ & $58.4(548)$ \\
\hline Single/divorced/widow, \% (n) & $37.4(351)$ & $39.1(367)$ \\
\hline \multicolumn{3}{|l|}{ Medical history } \\
\hline Depression, \% (n) & $13.3(125)$ & $13.9(131)$ \\
\hline Stroke, \% (n) & $3.5(33)$ & $3.5(33)$ \\
\hline Arthritis, \% (n) & $43.7(410)$ & $48.1(451)$ \\
\hline Diabetes, \% (n) & $20.5(192)$ & $21.5(202)$ \\
\hline Morbidity count, mean (SD) & $2.4(1.6)$ & $2.5(1.7)$ \\
\hline \multicolumn{3}{|l|}{ Medication history } \\
\hline Alpha-blocker, \% (n) & $6.7(63)$ & $5.2(49)$ \\
\hline Beta-blocker, \% (n) & $48.4(454)$ & $42.4(398)$ \\
\hline Diuretic, \% (n) & $29.6(278)$ & $23.8(223)$ \\
\hline Calcium antagonists, \% (n) & $43.9(412)$ & $37.5(352)$ \\
\hline $\begin{array}{l}\text { Angiotensin inhibitors/blockers, } \\
\%(n)\end{array}$ & $77.6(728)$ & $64.1(601)$ \\
\hline $\begin{array}{l}\text { Number of AHT classes, mean } \\
\text { (SD) }\end{array}$ & $2.1(1.0)$ & $2.1(0.9)$ \\
\hline AHT WHO-DDD, mean (SD) & $2.7(2.2)$ & $2.6(1.9)$ \\
\hline Antipsychotics, \% (n) & $2.8(26)$ & $2.0(19)$ \\
\hline Antidepressants, \% (n) & $15.6(146)$ & $13.5(127)$ \\
\hline Benzodiazepines, \% (n) & $9.2(86)$ & $7.2(68)$ \\
\hline NSAIDs, \% (n) & $9.1(85)$ & $6.0(56)$ \\
\hline Opiates, \% (n) & $6.2(58)$ & $5.7(53)$ \\
\hline Parkinson's disease, \% (n) & $1.1(10)$ & $1.3(12)$ \\
\hline Sedatives, \% (n) & $8.2(77)$ & $8.5(80)$ \\
\hline Urinary incontinence, \% (n) & $5.4(51)$ & $5.4(51)$ \\
\hline $\begin{array}{l}\text { Number of regular medicines, } \\
\text { mean (SD) }\end{array}$ & $6.2(3.7)$ & $5.9(4.1)$ \\
\hline
\end{tabular}

$\%$ may not add up to $100 \%$ due to missing data.

AHT, antihypertensive; NSAID, non-steroidal anti-inflammatory drug; WHO-DDD, WHO defined daily dose.

medication refill behaviour were not associated with injurious falls (aRR 1.04, 95\% CI 0.85 to $1.29, p=0.672$ ).

\section{Participant attrition}

There were no differences in injurious falls reported or the number of medication gaps between those included 
Table 2 The estimates, 95\% Cls and $p$ values for the association between 5-day gaps in antihypertensive medication adherence and injurious falls

\begin{tabular}{|c|c|c|c|c|c|c|}
\hline & Crude RR & $95 \% \mathrm{Cl}$ & $p$ values & Adj. RR & $95 \% \mathrm{Cl}$ & $p$ values \\
\hline Medication refill gaps $\geq 5$ days & 1.14 & 1.02 to 1.28 & 0.023 & 1.18 & 1.02 to 1.37 & 0.024 \\
\hline Age & 1.06 & 1.02 to 1.10 & 0.004 & 1.06 & 1.01 to 1.12 & 0.029 \\
\hline Female gender & 2.12 & 1.28 to 3.50 & 0.004 & 2.00 & 0.95 to 4.20 & 0.067 \\
\hline \multicolumn{7}{|l|}{ Education } \\
\hline Primary & Ref & - & - & - & - & - \\
\hline Secondary & 1.61 & 0.90 to 2.87 & 0.109 & 2.00 & 1.04 to 3.85 & 0.038 \\
\hline Third level & 1.41 & 0.70 to 2.83 & 0.331 & 1.54 & 0.66 to 3.55 & 0.315 \\
\hline \multicolumn{7}{|l|}{ Marital status } \\
\hline Married/partner & Ref & - & - & - & - & - \\
\hline Single/widow/div & 1.34 & 0.87 to 2.06 & 0.183 & 0.85 & 0.49 to 1.48 & 0.566 \\
\hline Depression & 0.87 & 0.39 to 1.94 & 0.737 & 0.25 & 0.08 to 0.84 & 0.025 \\
\hline Stroke & 0.74 & 0.19 to 2.90 & 0.666 & 1.01 & 0.18 to 5.67 & 0.990 \\
\hline Arthritis & 1.51 & 0.95 to 2.37 & 0.078 & 0.80 & 0.47 to 1.38 & 0.426 \\
\hline Diabetes & 0.73 & 0.39 to 1.35 & 0.312 & 0.71 & 0.35 to 1.44 & 0.346 \\
\hline Comorbidity count & 1.16 & 1.03 to 1.32 & 0.016 & 1.18 & 0.94 to 1.49 & 0.161 \\
\hline Alpha-blocker & 1.18 & 0.56 to 2.50 & 0.661 & 0.60 & 0.19 to 1.88 & 0.377 \\
\hline Beta-blocker & 1.17 & 0.77 to 1.77 & 0.461 & 1.29 & 0.76 to 2.19 & 0.346 \\
\hline Diuretics & 0.90 & 0.55 to 1.46 & 0.665 & 1.03 & 0.56 to 1.89 & 0.920 \\
\hline Calcium antagonists & 1.02 & 0.68 to 1.54 & 0.918 & 1.14 & 0.65 to 1.98 & 0.653 \\
\hline Angiotensin inhibitors/blockers & 0.73 & 0.45 to 1.19 & 0.212 & 0.84 & 0.46 to 1.54 & 0.576 \\
\hline Time since initial AHT Rx & 1.01 & 0.99 to 1.03 & 0.465 & 1.01 & 0.99 to 1.03 & 0.400 \\
\hline Antihypertensive WHO-DDD & 0.97 & 0.89 to 1.06 & 0.487 & 1.00 & 0.86 to 1.16 & 0.997 \\
\hline Addition/titration of $\mathrm{AHT}$ & 1.67 & 0.97 to 2.90 & 0.064 & 1.87 & 0.99 to 3.55 & 0.054 \\
\hline Antipsychotics & 0.94 & 0.23 to 3.80 & 0.933 & 1.52 & 0.34 to 6.75 & 0.581 \\
\hline Antidepressants & 1.55 & 0.92 to 2.63 & 0.102 & 1.80 & 0.87 to 3.74 & 0.113 \\
\hline Benzodiazepines & 2.03 & 1.20 to 3.44 & 0.008 & 1.29 & 0.62 to 2.68 & 0.493 \\
\hline NSAIDs & 0.55 & 0.22 to 1.39 & 0.208 & 0.52 & 0.19 to 1.44 & 0.207 \\
\hline Opiates & 2.28 & 1.27 to 4.09 & 0.006 & 2.00 & 1.06 to 3.76 & 0.032 \\
\hline Parkinsonian drugs & 2.49 & 0.70 to 8.85 & 0.158 & 2.97 & 0.44 to 20.0 & 0.263 \\
\hline Hypnotics and sedatives & 1.31 & 0.69 to 2.46 & 0.408 & 1.11 & 0.62 to 2.00 & 0.728 \\
\hline Urinary incontinence & 2.32 & 1.26 to 4.25 & 0.006 & 1.30 & 0.56 to 3.02 & 0.550 \\
\hline No of regular medicines & 1.08 & 1.03 to 1.14 & 0.003 & 1.06 & 0.98 to 1.15 & 0.139 \\
\hline
\end{tabular}

Modified Poisson regression with robust standard errors was used to estimate relative risks. Standard errors were adjusted for 104 clusters (pharmacy level). $n$ is smaller in final model $(n=724)$ due to missing data across covariates: medication refill gaps (7), age (5), education (46), marital status (31), medical history (1), medication history (6), antihypertensive WHO-DDD (16), addition/titration of AHT (156).

AHT, antihypertensive; NSAID, non-steroidal anti-inflammatory drug; RR, relative risk; WHO-DDD, WHO defined daily dose.

in the analysis compared with those lost to follow-up and excluded from the analysis. However, participants excluded due to attending other pharmacies tended to be younger, were less likely to report a heart attack and reported fewer comorbidities, while patients lost to follow-up at 12 months were older and used more regular medication, which resulted in a balance of these covariates in the final analysis.

\section{Missing data}

In the complete case multivariate analysis $(n=724)$ for the association between 5-day gaps in antihypertensive adherence and injurious, 22.8\% of observations were dropped due to missing data. The largest source of missing data was due to the variable indicating addition/titration of antihypertensive medication during follow-up ( $\mathrm{n}=156)$. These missing data were attributed to incomplete extraction of dispensing records at 12-month follow-up. Removing this variable from the final multivariate model yielded a model with a higher number of observations $(\mathrm{n}=856)$ and similar estimates for the association between 5-day gaps in adherence and injurious falls (aRR 1.16, 95\% CI 1.02 to 1.31, 


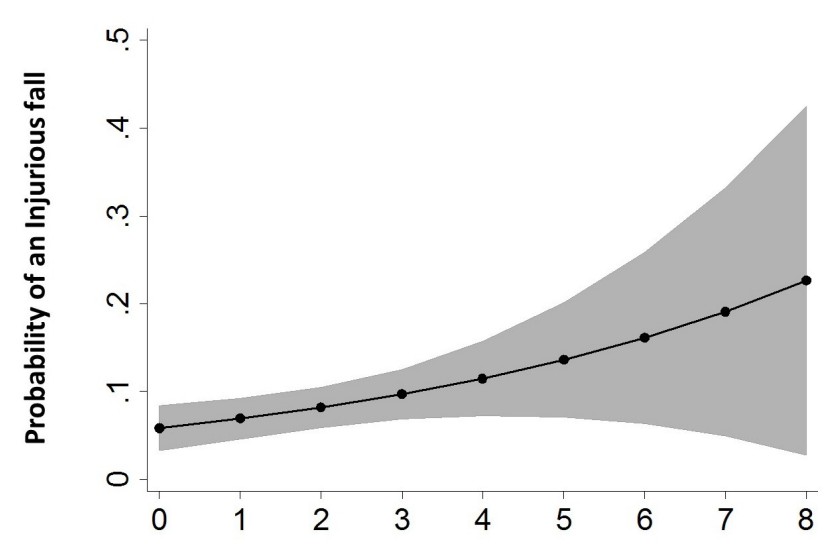

Number of 5-day medication refill gaps

Figure 2 Each 5-day gap in antihypertensive medication adherence was associated with an $18 \%$ increased risk of an injurious fall during follow-up (aRR 1.18, 95\% Cl 1.02 to 1.37 , $p=0.024)$. Wider Cls were observed at the upper end of the graph due to the low number of participants with six or more 5-day gaps in antihypertensive refill behaviour.

$p=0.019)$. Multiple imputation was also undertaken using a multivariate normal distribution, Markov Chain Monte Carlo procedure and 100 imputations. In this multiple imputation, similar estimates were obtained (aRR 1.15, $95 \%$ CI 1.02 to $1.30, p=0.020)$.

\section{DISCUSSION \\ Principal findings}

We observed an $18 \%$ increased risk of a self-reported injurious fall for each 5-day gap in antihypertensive medication refill adherence. This equated to an additional 11 injurious falls per 1000 patients with one 5-day gap in medication refill adherence compared with patients with no gaps. This finding was statistically significant following adjustment for a large number of clinically relevant fall risk factors including age, gender, comorbidities and fall risk increasing medication. Furthermore, a negative control exposure model did not indicate the potential for confounding due to the healthy adherer bias.

\section{Findings in the context of the previous literature}

This is the first study to examine medication adherence, specifically the quality of dose implementation on the risk of falls associated with antihypertensive medication use. A previous study by Berry et al identified an increased risk of falls for patients reporting lower medication adherence. They used a subjective method to evaluate medication adherence and did not differentiate adherence between drug classes. ${ }^{25}$ In contrast, we used an objective method to evaluate medication adherence and applied it to the exposure of interest, identifying that almost two-thirds of community-dwelling adults had at least one gap in antihypertensive medication refill over 12 months. A number of observational studies have consistently identified a higher risk of falling following antihypertensive medication initiation or titration. ${ }^{7-12}$ Patients with poor medication adherence, specifically poor implementation of antihypertensive pharmacotherapy, characterised by gaps in antihypertensive medication use, may experience a gradual rise in blood pressure. ${ }^{27-29}$ On resumption of therapy, similar to initial use, patients may be at a greater risk for falls relative to those who do not have gaps in antihypertensive therapy. In light of the consistent evidence indicating that initiating, titrating and adding antihypertensive medication are associated with a short-term increased risk of injurious falls, ${ }^{7-12}$ patients with gaps in their adherence to these therapies will potentially be exposed to similar pharmacological effects experienced on initiating antihypertensive medication. It has been suggested that the mechanism underlying falls risk during initiation of therapy is antihypertensive medication-induced acute orthostatic hypotension. ${ }^{5}$ Orthostatic hypotension is associated with an increased risk of injurious falls. ${ }^{1415}$ Further research is needed to investigate if gaps in antihypertensive medication adherence may also increase orthostatic hypotension.

There are conflicting findings on the association between falls and long-term antihypertensive use. Higher doses of antihypertensive medication ${ }^{1720}$ and the use of loop diuretics ${ }^{18}$ and non-selective beta-blockers ${ }^{21}{ }^{23}$ have been linked to falls, whereas a protective effect for the use of ACEIs and ARBs has been reported. ${ }^{19}$ Furthermore, two recent studies have found no association

Table 3 The adjusted regression models for the association between 5-day gaps in antihypertensive medication adherence and injurious falls from sensitivity analyses in (1) the negative control exposure analysis and (2) the weighted negative control exposure analysis

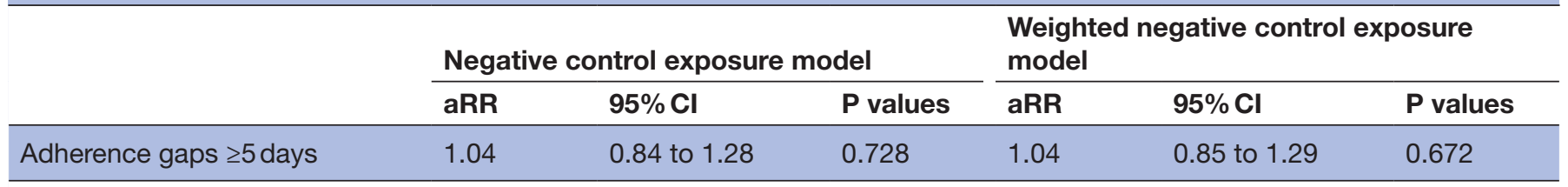

Modified Poisson regression with robust standard errors was used to estimate relative risks. Standard errors were adjusted for 104 clusters (pharmacy level). The estimates for the negative control exposure model $(n=515)$, tested the association between gaps in antithrombotic medication adherence and injurious falls, adjusted for covariates listed in table 1. A significant association in the negative exposure control model would indicate the presence of confounding associated with the exposure variable.

aRR, adjusted relative risk. 
between antihypertensive medication use and falls in older adults. ${ }^{162} \mathrm{~A}$ limitation to these studies is the failure to evaluate patients' medication adherence, potentially confounding findings. Chronic use of antihypertensive medication may improve systemic and cerebral haemodynamics and reduce orthostatic hypotension, leading to a reduction in injurious falls. ${ }^{14}{ }^{16}$ However, failing to account for medication adherence in long-term users may confound the association between antihypertensive use and injurious falls. Furthermore, medication adherence is known to vary according to the class of antihypertensive medication, with higher adherence associated with ACE inhibitors and ARBs, and lower adherence associated with diuretics and beta-blockers. ${ }^{44}$ These associations may be an explanatory factor for the protective effect observed for ACE inhibitors and ARBs, and the adverse effect observed for diuretics and beta-blockers on falls risk. Findings from randomised controlled trials of antihypertensive medication have reported no increase in falls risk. ${ }^{345-47}$ Nonetheless, trials tend to be of healthier populations that are not readily generalisable to real-world settings ${ }^{48}$ For example, the recent SPRINT trial demonstrated in adults $>75$ years that intensive blood pressure control was not associated with an increase in injurious falls. ${ }^{47}$ However, the external validity of this finding is questionable. A nationally representative longitudinal study of older adults meeting the SPRINT inclusion criteria observed a fivefold higher rate of injurious falls than the standard care group within SPRINT during a comparable follow-up period. ${ }^{49}$ Furthermore, it is apparent that medication adherence of clinical trial participants is higher. Two-thirds of our real-world participants had at least one 5-day gap in antihypertensive medication refill over a 12-month period compared with only half of the clinical trial participants who miss three consecutive days of antihypertensive within the same time period. ${ }^{26}$

\section{Strengths and limitations}

A strength of this study was the recruitment of community-dwelling older people from a national sample of pharmacies in Ireland, using a consecutive recruitment method. Although consecutive recruitment is a non-probabilistic method of sampling, it provides structured recruitment and additional rigour, ensuring all potential participants can be enrolled. Furthermore, the pharmacies included in this convenience sample are unlikely to differ significantly from other community pharmacies in Ireland. Only a small number of community pharmacies are continually involved in the NPIP, with approximately $40 \%-50 \%$ annual changeover in community pharmacies involved in the NPIP. These findings are likely to be only generalisable to those taking antihypertensive medication for $\geq 1$ year and who consistently attend the same pharmacy to obtain antihypertensive medication. Participants who we excluded because of attending other pharmacies were more likely to be younger and report less comorbidities. Additional strengths of this study include the prospective evaluation between medication gaps (measured prior to baseline) and injurious falls (evaluated from baseline to end of follow-up), with covariates remaining relatively consistent between baseline and follow-up. However, it was not possible to fully observe the temporal association between exposure and outcome, as the date of falls was not available. There are limitations to the use of a self-report measure of injurious falls, which may result in misclassification. Ideally, falls should be recorded prospectively on a daily basis with a minimum of monthly reporting, using methods such as postcards or diaries. However, these methods are resource intensive and are not feasible in many large-scale prospective studies. ${ }^{31}$ Less frequent gathering of falls data may result in misclassification in older people due to higher likelihood of memory impairment. However, a systematic review of studies comparing the validity of fall recall questionnaires with intensive prospective methods found falls recall to have high specificity $(91 \%-95 \%)$ and sensitivity $(80 \%-89 \%)$ in comparison with weekly postcards or daily diaries. ${ }^{31}$ Injurious falls are often recalled with greater accuracy due to resulting negative morbidity and subsequent medical treatment. ${ }^{50}$ Furthermore, we observed a comparable rate of injurious falls to a similar study of older adults in Ireland..$^{15}$ Excluding participants with possible cognitive impairment and dementia may have minimised fall recall misclassification. However, our methods to evaluate cognitive impairment may have resulted in misclassification due to potential underdiagnosis of patients in community settings. ${ }^{51}$ Although we excluded participants based on medication dispensed for cognitive impairment, it was not feasible during recruitment to screen all potential participants for cognitive impairment using a structured questionnaire. We also used an indirect method to evaluate medication adherence. Dispensing of medication from the pharmacy does not necessarily prove consumption. ${ }^{35}$ However, by evaluating medication refill adherence using a gap method, we can identify definitive periods where participants had at least 5 days during which they had insufficient medication to continuously consume antihypertensive medication until obtaining a subsequent supply. The resultant misclassification of medication adherence would weaken associations between medication refill gaps and injurious falls.

Additional strengths include the adjustment for a large number of potential confounders, including demographics, medical history and medication use. Furthermore, in comparison with other observational studies, medication use was evaluated objectively using pharmacy records, and concurrent changes to antihypertensive therapy including addition of classes and titration of doses were controlled for. Only established fall risk-increasing drugs were considered, hence, only specific drugs or classes identified in previous meta-analysis were included. ${ }^{38}$ Due to potential issues of testing a large number of predictors on a binary outcome with a low number of events in multivariate regression, a propensity score covariate adjustment model was also estimated, 
which did not change findings. ${ }^{40}$ The possibility for residual confounding may remain. Participants' blood pressure during the follow-up period was not measured to assess fluctuations in blood pressure. However, the ability of antihypertensive drugs to lower blood pressure have been proven in clinical trials, ${ }^{1-3}$ and clinical studies have shown that withdrawal of these medications leads to a gradual rebound in blood pressure. ${ }^{27-29}$ Furthermore, the feasibility of undertaking these measurements, which would likely require daily measurements, is unlikely in large observational studies such as this. Indeed measuring patient blood pressure on a daily basis would change the medication-taking behaviour of participants, known as white-coat adherence, which is similar to the Hawthorne effect. Some important fall risk factors were also unmeasured such as history of previous falls, frailty and disability. ${ }^{33}$ However, a negative control exposure analysis was undertaken to evaluate the potential for residual confounding. In the negative control model, no association between gaps in antithrombotic medication adherence and injurious falls was observed. Theoretically there should be no association and the negative control exposure model indicates that potential confounding resulting from the healthy adherer bias is unlikely. ${ }^{41} 42$

\section{CONCLUSION}

An 18\% increased risk of a self-reported injurious fall associated with each 5-day gap in antihypertensive medication refill adherence was observed. Our findings should be considered as hypothesis generating and future research should attempt to fully test the temporal relationship with injurious falls. If feasible, a direct method, such as electronic dose monitoring devices, should be used to evaluate the quality of dose implementation. Clinicians reconciling the risk of falls in older patients prescribed antihypertensive medication could use gaps in antihypertensive medication adherence as a marker to identify patients at higher risk of injurious falls. Although further research is needed to investigate whether improving adherence to antihypertensive medication can reduce injurious falls, advising patients to consistently take antihypertensive medication will reduce cardiovascular disease risk.

Acknowledgements The authors would like to acknowledge the Pharmaceutical Society of Ireland (PSI), for whom the Royal College of Surgeons in Ireland (RCSI) manages the National Pharmacy Internship Programme (NPIP). They also thank all Pharmacists who contributed to and facilitated the undertaking of this study.

Contributors PD, SMS, PJG, GC: involved in the conception and design of the study. PD and GC: undertook the acquisition and analysis of the work. PD, SMS, PJG, GC: interpreted the data. PD, SMS, PJG, GC: drafted the manuscript. PD, SMS, PJG, GC: revised the manuscript and gave final approval of the version to be published; agreed to be accountable for all aspects of the work in ensuring that questions related to the accuracy or integrity of any part of the work are appropriately investigated and resolved.

Funding The authors have not declared a specific grant for this research from any funding agency in the public, commercial or not-for-profit sectors.

Competing interests None declared.

Patient consent for publication Not required.
Ethics approval RCSI Research and Ethics Committee.

Provenance and peer review Not commissioned; externally peer reviewed.

Data sharing statement № additional data available.

Open access This is an open access article distributed in accordance with the Creative Commons Attribution Non Commercial (CC BY-NC 4.0) license, which permits others to distribute, remix, adapt, build upon this work non-commercially, and license their derivative works on different terms, provided the original work is properly cited, appropriate credit is given, any changes made indicated, and the use is non-commercial. See: http://creativecommons.org/licenses/by-nc/4.0/.

\section{REFERENCES}

1. Cushman WC, Davis BR, Pressel SL, et al. Mortality and morbidity during and after the Antihypertensive and Lipid-Lowering Treatment to Prevent Heart Attack Trial. J Clin Hypertens 2012;14:20-31.

2. Turnbull F, Neal B, Ninomiya $T$, et al. Effects of different regimens to lower blood pressure on major cardiovascular events in older and younger adults: meta-analysis of randomised trials. BMJ 2008;336:1121-3.

3. Wright JT, Williamson JD, Whelton PK, et al. A randomized trial of intensive versus standard blood-pressure control. N Engl J Med 2015;373:2103-16.

4. Berlowitz DR. Hypertension treatment and falls: should we be concerned? J Gen Intern Med 2014;29:1577-8.

5. Butt DA, Harvey PJ. Benefits and risks of antihypertensive medications in the elderly. J Intern Med 2015;278:599-626.

6. Berry SD, Kiel DP. Treating hypertension in the elderly: should the risk of falls be part of the equation? JAMA Intern Med 2014;174:596-7.

7. Gribbin J, Hubbard R, Gladman JR, et al. Risk of falls associated with antihypertensive medication: population-based case-control study. Age Ageing 2010;39:592-7.

8. Gribbin J, Hubbard R, Gladman J, et al. Risk of falls associated with antihypertensive medication: self-controlled case series. Pharmacoepidemiol Drug Saf 2011;20:879-84.

9. Butt DA, Mamdani M, Austin PC, et al. The risk of hip fracture after initiating antihypertensive drugs in the elderly. Arch Intern Med 2012;172:1739-44.

10. Butt DA, Mamdani M, Austin PC, et al. The risk of falls on initiation of antihypertensive drugs in the elderly. Osteoporos Int 2013;24:2649-57.

11. Shimbo D, Barrett Bowling C, Levitan EB, et al. Short-term risk of serious fall injuries in older adults initiating and intensifying treatment with antihypertensive medication. Circ Cardiovasc Qual Outcomes 2016;9:222-9.

12. Berry SD, Zhu $\mathrm{Y}$, Choi $\mathrm{H}$, et al. Diuretic initiation and the acute risk of hip fracture. Osteoporos Int 2013;24:689-95.

13. Kamaruzzaman $\mathrm{S}$, Watt $\mathrm{H}$, Carson $\mathrm{C}$, et al. The association between orthostatic hypotension and medication use in the British Women's Heart and Health Study. Age Ageing 2010;39:51-6.

14. Gangavati A, Hajjar I, Quach L, et al. Hypertension, orthostatic hypotension, and the risk of falls in a community-dwelling elderly population: the maintenance of balance, independent living, intellect, and zest in the elderly of Boston study. J Am Geriatr Soc 2011;59:383-9.

15. Finucane $\mathrm{C}, \mathrm{O}^{\prime}$ Connell MD, Donoghue $\mathrm{O}$, et al. Impaired orthostatic blood pressure recovery is associated with unexplained and injurious falls. J Am Geriatr Soc 2017;65:474-82.

16. Lipsitz LA, Habtemariam D, Gagnon M, et al. Reexamining the effect of antihypertensive medications on falls in old age. Hypertension 2015;66:183-9

17. Tinetti ME, Han L, Lee DS, et al. Antihypertensive medications and serious fall injuries in a nationally representative sample of older adults. JAMA Intern Med 2014;174:588-95.

18. Marcum ZA, Perera S, Newman AB, et al. Antihypertensive use and recurrent falls in community-dwelling older adults: findings from the health ABC Study. J Gerontol A Biol Sci Med Sci 2015;70:1562-8.

19. Wong AK, Lord SR, Sturnieks DL, et al. Angiotensin system-blocking medications are associated with fewer falls over 12 months in community-dwelling older people. J Am Geriatr Soc 2013;61:776-81.

20. Callisaya ML, Sharman JE, Close J, et al. Greater daily defined dose of antihypertensive medication increases the risk of falls in older people-a population-based study. J Am Geriatr Soc 2014;62:1527-33.

21. Ham AC, Swart KM, Enneman AW, et al. Medication-related fall incidents in an older, ambulant population: the B-PROOF study. Drugs Aging 2014;31:917-27. 
22. Richardson K, Bennett K, Kenny RA. Polypharmacy including falls risk-increasing medications and subsequent falls in communitydwelling middle-aged and older adults. Age Ageing 2015;44:90-6.

23. Ham AC, van Dijk SC, Swart KMA, et al. Beta-blocker use and fall risk in older individuals: Original results from two studies with metaanalysis. Br J Clin Pharmacol 2017;83:2292-302.

24. Vrijens B, De Geest S, Hughes DA, et al. A new taxonomy for describing and defining adherence to medications. Br J Clin Pharmacol 2012;73:691-705.

25. Berry SD, Quach L, Procter-Gray E, et al. Poor adherence to medications may be associated with falls. J Gerontol A Biol Sci Med Sci 2010;65:553-8.

26. Vrijens B, Vincze G, Kristanto P, et al. Adherence to prescribed antihypertensive drug treatments: longitudinal study of electronically compiled dosing histories. BMJ 2008;336:1114-7.

27. Myers MG, Leenen FH. General Practice Research Group. The impact of one or two missed doses on the duration of action of combined perindopril and indapamide. J Hum Hypertens 2007;21:86-93

28. Palatini P, Jung W, Shlyakhto E, et al. Maintenance of bloodpressure-lowering effect following a missed dose of aliskiren, irbesartan or ramipril: results of a randomized, double-blind study. $J$ Hum Hypertens 2010;24:93-103.

29. de Leeuw PW, Fagard R, Kroon AA. The effects of missed doses of amlodipine and losartan on blood pressure in older hypertensive patients. Hypertens Res 2017;40:568-72.

30. Kearney PM, Cronin H, O'Regan C, et al. Cohort profile: the Irish Longitudinal Study on Ageing. Int J Epidemiol 2011;40:877-84.

31. Ganz DA, Higashi T, Rubenstein LZ. Monitoring falls in cohort studies of community-dwelling older people: effect of the recall interval. $J$ Am Geriatr Soc 2005;53:2190-4.

32. Kardas P, Lewek P, Matyjaszczyk M. Determinants of patient adherence: a review of systematic reviews. Front Pharmacol 2013;4:91.

33. Deandrea S, Lucenteforte E, Bravi F, et al. Risk factors for falls in community-dwelling older people: a systematic review and metaanalysis. Epidemiology 2010;21:658-68.

34. Lamb SE, Jørstad-Stein EC, Hauer K, et al. Development of a common outcome data set for fall injury prevention trials: the Prevention of Falls Network Europe consensus. J Am Geriatr Soc 2005;53:1618-22.

35. Sattler EL, Lee JS, Perri M. Medication (re)fill adherence measures derived from pharmacy claims data in older Americans: a review of the literature. Drugs Aging 2013;30:383-99.

36. Giardini A, Martin MT, Cahir C, et al. Toward appropriate criteria in medication adherence assessment in older persons: Position Paper. Aging Clin Exp Res 2016;28:371-81.
37. Rang HP, Dale MM, Ritter JM, et al. Rang \& dale's pharmacology. 7th edn: Elsevier Churchill Livingstone, 2012.

38. Woolcott JC, Richardson KJ, Wiens MO, et al. Meta-analysis of the impact of 9 medication classes on falls in elderly persons. Arch Intern Med 2009;169:1952-60.

39. Zou GY, Donner A. Extension of the modified Poisson regression model to prospective studies with correlated binary data. Stat Methods Med Res 2013;22:661-70.

40. Franklin JM, Eddings W, Austin PC, et al. Comparing the performance of propensity score methods in healthcare database studies with rare outcomes. Stat Med 2017;36:1946-63.

41. Lipsitch M, Tchetgen Tchetgen E, Cohen T. Negative controls: a tool for detecting confounding and bias in observational studies. Epidemiology 2010;21:383-8.

42. LaFleur J, Nelson RE, Sauer BC, et al. Overestimation of the effects of adherence on outcomes: a case study in healthy user bias and hypertension. Heart 2011;97:1862-9.

43. Brookhart MA, Patrick AR, Shrank WH, et al. Validating studies of adherence through the use of control outcomes and exposures. Am $J$ Hypertens 2010;23:110.

44. Kronish IM, Woodward M, Sergie Z, et al. Meta-analysis: impact of drug class on adherence to antihypertensives. Circulation 2011;123:1611-21.

45. Peters R, Beckett N, Burch L, et al. The effect of treatment based on a diuretic (indapamide) +/- ACE inhibitor (perindopril) on fractures in the Hypertension in the Very Elderly Trial (HYVET). Age Ageing 2010;39:609-16.

46. Margolis KL, Palermo L, Vittinghoff E, et al. Intensive blood pressure control, falls, and fractures in patients with type 2 diabetes: the ACCORD trial. J Gen Intern Med 2014;29:1599-606.

47. Williamson JD, Supiano MA, Applegate WB, et al. Intensive vs standard blood pressure control and cardiovascular disease outcomes in adults aged $\geq 75$ years: a randomized clinical trial. JAMA 2016;315:2673.

48. Tinetti ME, Han L, McAvay GJ. Risks and benefits of antihypertensive medications in older adults-reply. JAMA Intern Med 2014:174:1873-4.

49. Sexton DJ, Canney M, O'Connell MDL, et al. Injurious falls and syncope in older community-dwelling adults meeting inclusion criteria for SPRINT. JAMA Intern Med 2017;177:1385-7.

50. Sanders KM, Stuart AL, Scott D, et al. Validity of 12-month falls recall in community-dwelling older women participating in a clinical trial. Int $J$ Endocrinol 2015;2015:1-6.

51. Connolly A, Gaehl E, Martin H, et al. Underdiagnosis of dementia in primary care: variations in the observed prevalence and comparisons to the expected prevalence. Aging Ment Health 2011;15:978-84. 